TRANSACTIONS OF THE

AMERICAN MATHEMATICAL SOCIETY

Volume 348, Number 6, June 1996

\title{
C*-ALGEBRAS WITH THE APPROXIMATE POSITIVE FACTORIZATION PROPERTY
}

\author{
G. J. MURPHY AND N. C. PHILLIPS
}

\begin{abstract}
We say that a unital $\mathrm{C}^{*}$-algebra $A$ has the approximate positive factorization property (APFP) if every element of $A$ is a norm limit of products of positive elements of $A$. (There is also a definition for the nonunital case.) T. Quinn has recently shown that a unital AF algebra has the APFP if and only if it has no finite dimensional quotients. This paper is a more systematic investigation of $\mathrm{C}^{*}$-algebras with the APFP. We prove various properties of such algebras. For example: They have connected invertible group, trivial $K_{1}$, and stable rank 1 . In the unital case, the $K_{0}$ group separates the tracial states. The APFP passes to matrix algebras, and if $I$ is an ideal in $A$ such that $I$ and $A / I$ have the APFP, then so does $A$. We also give some new examples of $\mathrm{C}^{*}$-algebras with the APFP, including type $\mathrm{II}_{1}$ factors and infinitedimensional simple unital direct limits of homogeneous $\mathrm{C}^{*}$-algebras with slow dimension growth, real rank zero, and trivial $K_{1}$ group. Simple direct limits of homogeneous $\mathrm{C}^{*}$-algebras with slow dimension growth which have the APFP must have real rank zero, but we also give examples of (nonsimple) unital algebras with the APFP which do not have real rank zero.

Our analysis leads to the introduction of a new concept of rank for a $\mathrm{C}^{*}$ algebra that may be of interest in the future.
\end{abstract}

\section{INTRODUCTION}

In recent years there has been some interest in the characterization of the operators on a Hilbert space that admit factorizations into products of "nice" operators, such as normal, selfadjoint, or positive operators. Quite different results are obtained depending on whether the dimension of the Hilbert space is finite or infinite. In the finite-dimensional case every operator is a product of two normal operators, by polar decomposition; an operator is a product of selfadjoint operators if and only if its determinant is real (H. Radjavi [23]); and an operator is a product of positive operators if and only if its determinant is nonnegative (C.S. Ballantine [1]). The infinite-dimensional case was analyzed by P.Y. Wu in [29]. There it is shown that for operators on a separable infinite-dimensional Hilbert space, the products of normal operators, of selfadjoint operators, and of positive operators all form the same class. Moreover, an operator belongs to this class if and only if it is a norm limit of invertible operators.

The study of factorization into positive and selfadjoint operators has been extended to several classes of $\mathrm{C}^{*}$-algebras: homogeneous $\mathrm{C}^{*}$-algebras (N.C. Phillips

Received by the editors April 21, 1994 and, in revised form, December 19, 1994.

1991 Mathematics Subject Classification. Primary 46L05, 46L10.

Partially supported by NSF grant DMS-9106285. 
[21]), and unitized stable $\mathrm{C}^{*}$-algebras and purely infinite simple $\mathrm{C}^{*}$-algebras (M. Leen $[15])$.

The question of approximate positive factorization was first raised for operators on Hilbert space, by M. Khalkhali, C. Laurie, B. Mathes, and H. Radjavi in [14]. This question has has been studied in a $\mathrm{C}^{*}$-algebra context by T. Quinn. In [22] he shows that if $A$ is an AF-algebra, then every element of $A$ is a norm limit of products of positive elements of $A$ if and only if $A$ admits no nonzero finitedimensional quotient $\mathrm{C}^{*}$-algebra.

In this paper we pursue the question of approximate positive factorization further. Our approach is new, involving additive commutators. Moreover, our emphasis is on a global as opposed to a local analysis; that is, we consider the question of determining global conditions on an algebra that ensure that all elements are approximable by products of positive elements. In this case, the algebra is said to have the approximate positive factorization property. (This is the definition for the unital case; that for the nonunital case is different. See below.) Our results lead to new classes of $\mathrm{C}^{*}$-algebras having the approximate positive factorization propertyfor example, type $\mathrm{II}_{1}$ factors, and infinite-dimensional simple unital direct limits with slow dimension growth, real rank zero, and trivial $K_{1}$ group.

$\mathrm{C}^{*}$-algebras having the approximate positive factorization property are shown to have some nice properties. For instance, it is shown in the unital case that the $K_{0}$ group separates the tracial states.

Our analysis leads to the introduction of a new concept of rank for a $\mathrm{C}^{*}$-algebra that may be of interest in the future.

The paper is organized as follows: In $\S 2$ we derive some properties of $\mathrm{C}^{*}$-algebras having the approximate positive factorization property. In $\S 3$ we consider some operations under which this class of $\mathrm{C}^{*}$-algebras is closed. In the final section, $\S 4$, we construct large classes of examples of $\mathrm{C}^{*}$-algebras with the approximate positive factorization property.

\section{The APPROXIMATE POSITIVE FACTORIZATION PROPERTY}

We begin by setting up some notation.

2.1. Notation. If $A$ is a unital $\mathrm{C}^{*}$-algebra, then $\operatorname{Inv}(A)$ is the invertible group of $A$, and $\operatorname{Inv}_{0}(A)$ is its identity component. Also, $U(A)$ is the unitary group of $A$ and $U_{0}(A)$ is its identity component.

If $A$ is a $\mathrm{C}^{*}$-algebra, then $\tilde{A}$ denotes its unitization (with a new unit adjoined even if $A$ already has one), and $A+1$ denotes $\{a \in \tilde{A}: a-1 \in A\}$. If $A$ is nonunital, we define $\operatorname{Inv}(A)=\operatorname{Inv}(\tilde{A}) \cap(A+1)$ and $\operatorname{Inv}_{0}(A)=\operatorname{Inv}_{0}(\tilde{A}) \cap(A+1)$, and define $U(A)$ and $U_{0}(A)$ similarly.

If $A$ is unital, then there is a canonical isomorphism $\tilde{A} \cong A \oplus \mathbf{C}$. If in this case we identify an invertible element $a \in A$ with $(a, 1) \in A \oplus \mathbf{C}$, then the definitions given for the nonunital case, when applied to unital $\mathrm{C}^{*}$-algebras, agree with the ones for the unital case.

2.2. Definition. Let $A$ be a unital $\mathrm{C}^{*}$-algebra. Let $P(A)$ denote the set of finite products of positive elements of $A$. We say that $A$ has the approximate positive factorization property if $P(A)$ is dense in $A$. If $A$ is not unital, then we say $A$ has the approximate positive factorization property if the set $P(A+1)$ of finite products of positive elements in $A+1$ is dense in $A+1$. 
If $A$ is unital, one easily checks that $P(A)^{-}=A$ if and only if $P(A+1)^{-}=A+1$. Thus, we can (and will) prove results for both the unital and nonunital cases using only the setup for the nonunital case.

It will be clear from results below that a unitization $\tilde{A}$ can never have the approximate positive factorization property. It is, however, not at all clear what the relation is between our definition for nonunital $\mathrm{C}^{*}$-algebras and the condition that products of positive elements of $A$ be dense in $A$. We use the definition above because it works in our theorems.

We make a few comments on $P(A)$. Obviously, it is multiplicatively closed. Also, it is invariant under similarity; that is, if $v$ is an invertible element of $A$, then $v P(A) v^{-1}=P(A)$. (This is easily seen by polar decomposing $v$ into a product of a unitary element and a positive element.) Finally, we note that one sided invertibility implies invertibility for positive elements, so that if $a=a_{1} \cdots a_{n} \in P(A)$, with $a_{1}, \ldots, a_{n}$ positive, and if $a$ is invertible, then so are the $a_{j}$.

In Theorem 2.4 below, we give some consequences of the approximate positive factorization property. We need the following lemma. It is the analog of the fact that if $A$ has stable rank 1 , then so do all matrix algebras over $A$. We denote by $M_{n}(A)$ the algebra of $n \times n$ matrices over $A$, and write $M_{n}$ when $A=\mathbf{C}$.

2.3. Lemma. Let $A$ be a unital $C^{*}$-algebra in which $\operatorname{Inv}_{0}(A)$ is dense. Then $\operatorname{Inv}_{0}\left(M_{n}(A)\right)$ is dense in $M_{n}(A)$ for each positive integer $n$.

Proof. We prove the result by induction on $n$. If $x$ is an element of $M_{n}(A)$, we may write it in the form

$$
x=\left(\begin{array}{ll}
a & b \\
c & d
\end{array}\right),
$$

where $a \in M_{n-1}(A), d \in A$ and $b$ and $c$ are appropriate column and row matrices with entries in $A$. To show that $x \in\left(\operatorname{Inv}_{0}\left(M_{n}(A)\right)\right)^{-}$, we may suppose that $d$ belongs to $\operatorname{Inv}_{0}(A)$, since, by assumption, $\operatorname{Inv}_{0}(A)$ is dense in $A$. Hence, we may set $y=a-b d^{-1} c$. Then

$$
x=\left(\begin{array}{cc}
1 & b d^{-1} \\
0 & 1
\end{array}\right)\left(\begin{array}{ll}
y & 0 \\
0 & d
\end{array}\right)\left(\begin{array}{cc}
1 & 0 \\
d^{-1} c & 1
\end{array}\right) .
$$

The second factor is in $\left(\operatorname{Inv}_{0}\left(M_{n}(A)\right)\right)^{-}$by the inductive hypothesis, since $y \in$ $\left(\operatorname{Inv}_{0}\left(M_{n-1}(A)\right)\right)^{-}$. The other two factors are clearly in $\operatorname{Inv}_{0}\left(M_{n}(A)\right)$. So $x \in$ $\left(\operatorname{Inv}_{0}\left(M_{n}(A)\right)\right)^{-}$.

2.4. Theorem. Let $A$ be any $C^{*}$-algebra (unital or not) with the approximate positive factorization property. Then:

(1) $\operatorname{Inv}\left(M_{n}(A)\right)$ is connected for all $n$.

(2) $K_{1}(A)=0$.

(3) The (topological) stable rank $\operatorname{sr}(A)$ is 1 .

(4) The connected stable rank $\operatorname{csr}(A)([24]$, Definition 4.7) is 1.

Proof. To avoid repetition, we use the setup for the nonunital case.

Functional calculus shows that every positive element in $A+1$ is a limit of positive invertible elements in $A+1$. Such elements are clearly in $\operatorname{Inv}_{0}(A)$. Therefore $\operatorname{Inv}_{0}(A)$ is dense in $A+1$. By factoring out scalars, we see that $\operatorname{Inv}_{0}(\tilde{A})$ is dense in $\tilde{A}$. This proves (3), and (1) now follows from the previous lemma. (Note that $\operatorname{Inv}(B)$ is 
connected if and only if $\operatorname{Inv}(\tilde{B})$ is connected.) Conclusion (2) is immediate from (1). For (4), we note that, by the remarks after Corollary 4.10 of [24], it suffices to show that left invertible elements in $A$ are invertible and that $\operatorname{Inv}(A)$ is connected. The first statement follows from (3) and the second from (1).

If $A$ is any unital $\mathrm{C}^{*}$-algebra, we denote by $G(A)$ the set of all elements $\lambda \in \mathbf{T}$, where $\mathbf{T}$ denotes the set of unit-modulus scalars, such that $\lambda 1 \in P(A)^{-}$. Clearly, $G(A)$ is a closed subgroup of $\mathbf{T}$, and therefore it is the group of all $n$th roots of unity, for some positive integer $n$, or it is equal to $\mathbf{T}$.

A number of concepts of rank for $\mathrm{C}^{*}$-algebras have been introduced in recent years, such as the real, analytic, and stable ranks. (See $[3,17,24]$.) Using the group $G(A)$ we can define another rank function. It will be technically useful here, and may be of independent interest in the future.

2.5. Definition. We set $\operatorname{rank}(A)=n$ if $G(A)$ is finite with $n$ elements, and we set $\operatorname{rank}(A)=\infty$ if $G(A)=\mathbf{T}$.

2.6. Proposition. The function rank has the following properties for unital $C^{*}$-algebras.

(1) $\operatorname{rank}\left(M_{n}\right)=n$.

(2) The algebra $B(H)$ of bounded operators on a separable infinite-dimensional Hilbert space $H$ satisfies $\operatorname{rank}(B(H))=\infty$.

(3) If $\varphi: A \rightarrow B$ is a unital homomorphism, then $\operatorname{rank}(A)$ divides $\operatorname{rank}(B)$. (Any integer is considered to divide $\infty$.) In particular, if $B$ is a unital $C^{*}$-algebra and $A$ is a $C^{*}$-subalgebra of $B$ containing the identity of $B$, then $\operatorname{rank}(A)$ divides $\operatorname{rank}(B)$.

(4) If $A$ has a nonzero representation on $\mathbf{C}^{n}$, then $\operatorname{rank}(A) \leq n$.

(5) If $\operatorname{rank}(A)>1$, then the commutator ideal of $A$ is all of $A$.

Proof. Parts (1) and (2) follow from Ballantine's theorem [1] and Wu's characterization of $P(B(H))$ [29] respectively. For (3), note that $\varphi(G(A))$ is a subgroup of $G(B)$. Part (4) is immediate from part (3), and part (5) follows since if the commutator ideal is proper, then $A$ has a one-dimensional representation.

If $X$ is a compact Hausdorff space, then $M_{n} \otimes C(X)$ has rank $n$. This follows from [21], and is also easy to check directly.

We also observe that a unital $\mathrm{C}^{*}$-algebra $A$ with infinite rank can never be of type I. Indeed, if $A$ is unital and of type I, choose a maximal ideal $M$ of $A$, and note that $A / M \cong M_{n}$ for some $n<\infty$. Thus, $\operatorname{rank}(A) \leq n$.

A unital $\mathrm{C}^{*}$-algebra with the approximate positive factorization property has infinite rank, so we obtain:

2.7. Proposition. Let $A$ be a unital $C^{*}$-algebra with the approximate positive factorization property. Then:

(1) A has no nonzero finite-dimensional representations.

(2) The commutator ideal of $A$ is all of $A$.

We next establish a connection between the approximate positive factorization property and the behavior on the $K_{0}$-group of tracial states. We will need part (2) of the following lemma; part (1) will be used at the end of this section. 
2.8. Lemma. Let $A$ be a unital $C^{*}$-algebra, let $\tau: A \rightarrow \mathbf{C}$ be a selfadjoint (but not necessarily positive) bounded linear tracelike functional, and let $a \in A$ be selfadjoint. Then:

(1) If $\exp (2 \pi i a) \in P(A)$ then $\tau(a) \in \tau_{*}\left(K_{0}(A)\right)$.

(2) If $\exp (2 \pi i a) \in P(A)^{-}$then $\tau(a) \in \tau_{*}\left(K_{0}(A)\right)^{-}$.

Proof. The proofs of the two parts are similar, but the proof of (2) is slightly more complicated. Therefore we only prove (2).

Clearly we may assume $\tau \neq 0$. Let $\varepsilon>0$. Use polar decomposition to choose positive elements $b_{1}, \ldots, b_{n} \in A$ such that $z=b_{1} \cdots b_{n}$ is unitary and $\|z-\exp (2 \pi i a)\|<$ $2 \pi \varepsilon /\|\tau\|$. Set $h=\frac{1}{2 \pi i} \log \left(\exp (2 \pi i a)^{*} z\right)$. Then $h$ is selfadjoint and satisfies $z=$ $\exp (2 \pi i a) \exp (2 \pi i h)$ and $\|h\|<\varepsilon /\|\tau\|$. Let $\Delta$ be the de la Harpe-Skandalis determinant [8] associated to $\tau$; it is a homomorphism from $\operatorname{Inv}_{0}(A)$ to the additive group $\mathbf{C} / \tau_{*}\left(K_{0}(A)\right)$. Proposition $2(\mathrm{~b})$ of $[8]$ states that $\Delta(\exp (c))=\frac{1}{2 \pi i} \tau(c)+\tau_{*}\left(K_{0}(A)\right)$ for any $c \in A$. Computing $\Delta(z)$ in two different ways, we obtain

$$
\frac{1}{2 \pi i} \sum_{k=1}^{n} \tau\left(\log \left(b_{k}\right)\right)-\tau(a)-\tau(h) \in \tau_{*}\left(K_{0}(A)\right) .
$$

(Note that $\log \left(b_{k}\right)$ is defined since $b_{k}$ is invertible.) Thus, we can write

$$
\frac{1}{2 \pi i} \sum_{k=1}^{n} \tau\left(\log \left(b_{k}\right)\right)=\tau(a)+\tau(h)-\alpha
$$

for some $\alpha \in \tau_{*}\left(K_{0}(A)\right)$. The right hand side of this equation is real and the left hand side is purely imaginary. Therefore both sides are zero. Since $|\tau(h)| \leq$ $\|\tau\|\|h\|<\varepsilon$, we get $|\tau(a)-\alpha|<\varepsilon$. Thus $\operatorname{dist}\left(\tau(a), \tau_{*}\left(K_{0}(A)\right)\right)<\varepsilon$. Since $\varepsilon>0$ is arbitrary, this shows that $\tau(a) \in \tau_{*}\left(K_{0}(A)\right)^{-}$.

As a corollary, we obtain the following theorem.

2.9. Theorem. Let $A$ be a unital $C^{*}$-algebra with the approximate positive factorization property. Then $K_{0}(A)$ distinguishes the tracial states on $A$. That is, if $\tau_{1}$ and $\tau_{2}$ are two tracial states on $A$ such that $\left(\tau_{1}\right)_{*}=\left(\tau_{2}\right)_{*}$ as maps from $K_{0}(A)$ to $\mathbf{R}$, then $\tau_{1}=\tau_{2}$.

Proof. Suppose $K_{0}(A)$ does not distinguish the tracial states on $A$. Let $\tau_{1}$ and $\tau_{2}$ be two distinct tracial states such that $\left(\tau_{1}\right)_{*}=\left(\tau_{2}\right)_{*}$. Set $\tau=\tau_{1}-\tau_{2}$. Then $\tau_{*}\left(K_{0}(A)\right)=\{0\}$. Since $\tau_{1} \neq \tau_{2}$, there is a selfadjoint $a \in A$ such that $\tau_{1}(a) \neq \tau_{2}(a)$. Then $\exp (2 \pi i a) \notin P(A)^{-}$by part (2) of the previous lemma.

For some classes of $\mathrm{C}^{*}$-algebras (see [2] and Theorem 4.9 below), the conclusion of this theorem implies that the algebra has real rank zero [3]. Example 4.16 below will show, however, that the approximate positive factorization property does not in general imply real rank zero.

Lemma 2.8(1) yields results on $P(A)$ which show why one should consider the condition $P(A)^{-}=A$ rather than $P(A)=A$.

2.10. Proposition. Let $A$ be a separable unital $C^{*}$-algebra which has a tracial state $\tau$. (Equivalently, the linear span of the commutators $a b-b a$ is not dense.) Then there is $\lambda \in \mathbf{C}$ such that $\lambda \cdot 1 \notin P(A)$. 
Proof. Since $A$ is separable, $K_{0}(A)$ is countable, so $\tau_{*}\left(K_{0}(A)\right)$ is a proper subset of R. Choose $\alpha \in \mathbf{R}$ such that $\alpha \notin \tau_{*}\left(K_{0}(A)\right)$. Then $\exp (2 \pi i \alpha) \cdot 1 \notin P(A)$ by Lemma 2.8(1).

2.11. Remark. This proposition shows that if $A$ is a simple separable unital stably finite exact $\mathrm{C}^{*}$-algebra, then there is $\lambda \in \mathbf{C}$ such that $\lambda \cdot 1 \notin P(A)$. Indeed, it follows from Theorem 6.1 of [27] that a simple stably finite $\mathrm{C}^{*}$-algebra has a nontrivial quasitrace. By [11], every quasitrace on an exact $\mathrm{C}^{*}$-algebra is a trace.

We can even use Lemma 2.8 to shed some more light on the rank defined above:

2.12. Proposition. Let $A$ be a unital $C^{*}$-algebra. Suppose $n$ is a positive integer and $\tau$ is a tracial state on $A$ such that $n \tau_{*}\left(K_{0}(A)\right) \subset \mathbf{Z}$. Then $\operatorname{rank}(A) \mid n$.

Proof. The hypotheses imply that $\tau_{*}\left(K_{0}(A)\right)$ is closed in $\mathbf{R}$. Lemma 2.8(2) therefore implies that if $\alpha \in \mathbf{R}$ and $\exp (2 \pi i \alpha) \cdot 1 \in P(A)^{-}$, then $n \alpha \in \mathbf{Z}$. Thus $G(A)$ is a subgroup of $\left\{\zeta \in \mathbf{T}: \zeta^{n}=1\right\}$.

2.13. Proposition. If $A$ is unital and has infinite rank, then $\tau_{*}\left(K_{0}(A)\right)$ is dense in $\mathbf{R}$ for any tracial state $\tau$.

Proof. This is immediate from Lemma 2.8(2).

This proposition shows that if $A$ has a tracial state and has infinite rank, then $K_{0}(A)$ must be large. Note, however, that the Cuntz algebra $\mathcal{O}_{2}$ has infinite rank (since, by [15], $\operatorname{Inv}_{0}(A) \subset P(A)$ for purely infinite simple unital $\mathrm{C}^{*}$-algebras $A$ ), but $K_{0}\left(\mathcal{O}_{2}\right)=0$.

2.14. Example. Let $C_{\mathrm{r}}^{*}\left(F_{m}\right)$ denote the reduced $\mathrm{C}^{*}$-algebra of the free group on $m$ generators. Then $\operatorname{rank}\left(M_{n} \otimes C_{\mathrm{r}}^{*}\left(F_{m}\right)\right)=n$. Indeed, $\operatorname{rank}\left(M_{n} \otimes C_{\mathrm{r}}^{*}\left(F_{m}\right)\right) \geq n$ by Proposition 2.6(1) and (3), applied to the subalgebra $M_{n} \otimes 1$. The reverse inequality follows from Proposition 2.12.

The same argument shows that if $A$ is any unital $\mathrm{C}^{*}$-algebra with a tracial state $\tau$ for which $\tau_{*}\left(K_{0}(A)\right)=\mathbf{Z}$, then $\operatorname{rank}\left(M_{n} \otimes A\right)=n$.

This example gives simple infinite-dimensional $\mathrm{C}^{*}$-algebras $A$ with arbitrary finite values of $\operatorname{rank}(A)$. On the other hand, Theorem 4.10 below gives many stably finite simple unital $\mathrm{C}^{*}$-algebras with infinite rank.

\section{INVARIANCE PROPERTIES}

In this section, we prove that the approximate positive factorization property is preserved under several natural operations on $\mathrm{C}^{*}$-algebras. Our first goal is to show that if $A$ has the approximate positive factorization property, then so does $M_{n}(A)$. The following notation will be useful. To avoid repetition, we work in the setup for the nonunital case.

3.1. Notation. Let $A$ be a $\mathrm{C}^{*}$-algebra. We denote by $[A, A]$ the closed linear span of the commutators $[a, b]=a b-b a$ of $A$ and by $L(A)$ the set of all elements $a$ of $A$ such that $\exp (\lambda a)$ belongs to $P(A+1)^{-}$for all $\lambda \in \mathbf{C}$.

Note that $[\tilde{A}, \tilde{A}]=[A, A]$, and that if $a \in A$ then $\exp (a)$ is always in $A+1$. Further, if $A$ is unital, $a \in A$, and $\exp (a)$, calculated in $\tilde{A}$, is in $P(A+1)^{-}$, then $\exp (a)$, calculated this time in $A$, is in $P(A)^{-}$. Also, it is obvious that if $L(A)=A$ and $\operatorname{Inv}_{0}(\tilde{A})$ is dense in $\tilde{A}$, then $P(A+1)$ is dense in $A+1$, and conversely. 
Before proceeding, we need to recall some known results about exponentials. If $a, b \in A$, then

$$
\exp (a+b)=\lim _{n \rightarrow \infty}(\exp (a / n) \exp (b / n))^{n}
$$

and

$$
\exp ([a, b])=\lim _{n \rightarrow \infty}(\exp (-a / n) \exp (-b / n) \exp (a / n) \exp (b / n))^{n^{2}} .
$$

For lack of a better source, we refer to the proof of Lemma 2.4 of [20].

3.2. Lemma. Let $A$ be a $C^{*}$-algebra. Then $L(A)$ is a closed, linear subspace of $A$ containing $[A, A]$.

Proof. It is obvious that $L(A)$ contains 0 , is norm closed, and is closed under multiplication by scalars. That $L(A)$ is closed under addition follows from equation (1). To see that $[A, A] \subseteq L(A)$, it suffices to show that $[a, b] \in L(A)$ for all $a, b \in A$. Hence, it is sufficient to show that $\exp ([a, b]) \in P(A+1)^{-}$if $a, b \in A$ and $b=b^{*}$. For such elements $a, b$ we have, by equation (2),

$$
\exp ([a, b])=\lim _{n \rightarrow \infty}\left(v_{n} \exp (-b / n) v_{n}^{-1} \exp (b / n)\right)^{n^{2}},
$$

where $v_{n}=\exp (-a / n)$. Since $\exp (b / n)$ is positive and since $P(A+1)$ is invariant under similarity, we have $v_{n} \exp (-b / n) v_{n}^{-1} \exp (b / n) \in P(A+1)$ and therefore $\exp ([a, b]) \in P(A+1)^{-}$, as required.

3.3. Example. Let $H$ be an infinite-dimensional Hilbert space, and let $A=$ $B(H)$. Since every operator on $H$ is a sum of two commutators ([12], Corollary 2 to Problem 234), we have $[A, A]=A$. Hence, $\exp (a) \in P(A)^{-}$for all $a \in A$, by the previous lemma. Of course, $\operatorname{Inv}_{0}(A)=\operatorname{Inv}(A)$ in this case, so $P(A)^{-}=$ $(\operatorname{Inv}(A))^{-}$. This generalizes, for if $A$ is any properly infinite von Neumann algebra, then $[A, A]=A[18]$ and therefore $P(A)^{-}=(\operatorname{Inv}(A))^{-}$, again by the previous lemma.

3.4. Theorem. Let $A$ be a $C^{*}$-algebra having the approximate positive factorization property. Then for each positive integer $n$, the matrix algebra $M_{n}(A)$ also has the approximate positive factorization property.

Proof. We first show that if $a=\left(a_{i j}\right)$ is an element of $M_{n}(A)$ with zero diagonal, then $a$ is a limit of sums of commutators. To do this we identify $M_{n}(A)$ with the tensor product $M_{n} \otimes A$ in the usual way, so that, if the elements $e^{i j}(i, j=1, \ldots, n)$ form the canonical basis for $M_{n}$, we may write $a=\sum_{i, j} e^{i j} \otimes a_{i j}$. Let $\left(e_{\lambda}\right)$ be an approximate identity for $A$. Then the equation

$$
a=\lim _{\lambda} \sum_{i, j}\left[e^{i j} \otimes a_{i j}, e^{j j} \otimes e_{\lambda}\right]
$$

expresses $a$ as a limit of sums of commutators.

Now let $a$ be an arbitrary element of $M_{n}(A)$. Clearly, $a=b+c$, where $b$ has zero diagonal and $c$ is a diagonal matrix. Since $b$ is a limit of sums of commutators, $b$ belongs to $L\left(M_{n}(A)\right)$ by Lemma 3.2, and since $L(A)=A$ it is clear that $c$ belongs to $L\left(M_{n}(A)\right)$. Hence, $L\left(M_{n}(A)\right)=M_{n}(A)$. Moreover, since $\left(\operatorname{Inv}_{0}(\tilde{A})\right)^{-}=\tilde{A}$, we have $\left(\operatorname{Inv}_{0}\left(M_{n}(\tilde{A})\right)\right)^{-}=M_{n}(\tilde{A})$ by Lemma 2.3. Combining these two facts, we get $P\left(M_{n}(A)+1\right)^{-}=M_{n}(A)+1$. 
3.5. Theorem.

(1) The finite direct sum of $C^{*}$-algebras with the approximate positive factorization property has the approximate positive factorization property.

(2) The direct limit of $C^{*}$-algebras with the approximate positive factorization property has the approximate positive factorization property.

(3) If $A$ has the approximate positive factorization property and $F$ is a finitedimensional $C^{*}$-algebra, then $A \otimes F$ has the approximate positive factorization property.

(4) If $A$ has the approximate positive factorization property and $F$ is an $A F$ algebra, then $A \otimes F$ has the approximate positive factorization property.

Proof. Parts (1) and (2) are immediate. Part (3) follows from (1) and the previous theorem, and part (4) follows from (2) and (3).

3.6. Theorem. Let $A$ be a $C^{*}$-algebra, and let $I$ be a closed ideal in $A$. Then:

(1) If $I$ and $A / I$ have the approximate positive factorization property, then so does $A$.

(2) If $A$ has the approximate positive factorization property, then so does $A / I$.

Proof. Let $B=A / I$ and let $\pi: \tilde{A} \rightarrow \tilde{B}$ be the quotient map.

(1) Theorem 2.4(3) and (4), and Theorem 4.11 of [24], imply that $\operatorname{sr}(A)=1$. By polar decomposition, it therefore suffices to prove that $U(\tilde{A}) \cap(A+1) \subset P(A+1)^{-}$. Let $u \in U(\tilde{A}) \cap(A+1)$, and let $\varepsilon>0$. Continuity of the polar decomposition allows us to use the approximate positive factorization property in $B$ to find a unitary $y \in P(B+1)$ such that $\|y-\pi(u)\|<\min (1, \varepsilon / 2)$.

Write $y=b_{1} \cdots b_{m}$ with $b_{1}, \ldots, b_{m} \in B+1$ positive. Then the $b_{j}$ are also invertible. Hence for each $j$ there is $\rho_{j}>-1$ such that $\operatorname{sp}\left(b_{j}-1\right) \subset\left[\rho_{j}, \infty\right)$. Using functional calculus, it is easy to choose $a_{j} \in A$ selfadjoint such that $\pi\left(a_{j}\right)=b_{j}-1$ and $\operatorname{sp}\left(a_{j}\right) \subset\left[\rho_{j}, \infty\right)$. In particular, $a_{j}+1$ is invertible. Set $x_{0}=\left(a_{1}+1\right) \cdots\left(a_{m}+1\right)$, and let $x$ be the unitary part of the polar decomposition of $x_{0}$. Then $x \in P(A+1)$ and $\pi(x)=y$. Therefore $\left\|\pi\left(x^{*} u-1\right)\right\|<\varepsilon / 2$. Clearly also $x^{*} u-1 \in A$.

Choose $c_{0} \in A$ such that $\pi\left(c_{0}\right)=\pi\left(x^{*} u-1\right)$ and $\left\|c_{0}\right\|<\varepsilon / 2$. Set $c=x^{*} u-c_{0}$. Then $\pi(c)=1$, so that $c \in I+1$. Therefore there is $r \in P(I+1)$ such that $\|r-c\|<\varepsilon / 2$. It follows that $x r \in P(A+1)$ and $\|x r-u\| \leq\|r-c\|+\left\|c_{0}\right\|<\varepsilon$.

(2) This is immediate from the fact that $\pi(A+1)=B+1$.

We will see below (Example 4.16) that the approximate positive factorization property does not always pass to ideals.

\section{Characterizations AND Classes of EXAmples}

In this section, we construct a variety of examples of $\mathrm{C}^{*}$-algebras having the approximate positive factorization property. In some special classes we can even give necessary and sufficient conditions for the approximate positive factorization property.

We begin by recalling the following theorem of Quinn [22]:

4.1. Theorem. A unital AF algebra has the approximate positive factorization property if and only if it has no nonzero finite-dimensional quotient $C^{*}$-algebras.

Next, we consider von Neumann factors. We need two lemmas. 
4.2. Lemma. Let $A$ be a unital $C^{*}$-algebra. Then $[A, A]+\mathbf{C} 1=A$ if and only if $A$ has at most one tracial state.

Proof. This follows from Proposition 2.7 of [7], using Theorem 2.6 and the definitions in Section 2 of the same paper.

A simpler proof than that of [7] can be obtained as follows. The forward implication is obvious. As for the reverse implication, if $[A, A]+\mathbf{C} 1 \neq A$, then the Hahn-Banach theorem can be used to find a nonzero selfadjoint bounded linear functional which vanishes on $[A, A]+\mathbf{C} 1$, and the Jordan decomposition then yields two different tracial states.

4.3. Lemma. Let $A$ be a unital $C^{*}$-algebra such that $[A, A]+\mathbf{C} 1=A$ and such that $\operatorname{Inv}_{0}(A)$ is dense in $A$. Then for each element $a \in A$ there exists $\lambda \in \mathbf{T}$ such that $\lambda a \in P(A)^{-}$.

Proof. If $a \in A$, then for some scalar $\lambda$ the element $a-\lambda$ belongs to $[A, A]$ and therefore $\exp (a-\lambda)$ belongs to $P(A)^{-}$, by Lemma 3.2. Hence, $\lambda^{\prime} \exp (a) \in P(A)^{-}$, where $\lambda^{\prime}=\exp (-\lambda) /|\exp (-\lambda)|$ and $\left|\lambda^{\prime}\right|=1$. It is an immediate consequence that for each element $a$ of $\operatorname{Inv}_{0}(A)$ there exists $\lambda \in \mathbf{T}$ such that $\lambda a \in P(A)^{-}$. That the same result therefore holds for any element $a$ of $A$ now follows from the assumption that $\operatorname{Inv}_{0}(A)$ is dense in $A$.

4.4. Theorem. A factor has the approximate positive factorization property if and only if it is of type $\mathrm{II}_{1}$.

Proof. Let $A$ be a factor with the approximate positive factorization property. Then $\operatorname{sr}(A)=1$, so $A$ is finite. Proposition 2.7(1) implies that $A$ is infinite-dimensional. Hence, $A$ must be of type $\mathrm{II}_{1}$.

Now suppose conversely that $A$ is of type $\mathrm{II}_{1}$. Then it is simple and has a unique tracial state, since, as is well known, this is true of any finite factor. Consequently, by Lemma $4.2,[A, A]+\mathbf{C} 1=A$. Moreover, by a result of $\mathrm{H}$. Choda [4], finiteness of $A$ implies $\operatorname{Inv}_{0}(A)$ is dense in $A$. Hence, by the previous lemma, for each element $a$ of $A$, there exists a scalar $\lambda$ of unit modulus such that $\lambda a$ belongs to $P(A)^{-}$. To complete the proof it is clear that we need now show only that $\operatorname{rank}(A)=\infty$. It is well known that a type $\mathrm{II}_{1}$ factor contains a unital copy of $M_{n}$ for any $n$, and it therefore follows from Ballantine's theorem [1] that $G(A)$ contains all $n$th roots of unity for every $n$. Therefore $G(A)=\mathbf{T}$.

4.5. Example. The previous theorem, combined with the method of Example 3.10 of [19], yields a simple separable nonnuclear (hence not AF) unital $\mathrm{C}^{*}$-algebra with the approximate positive factorization property.

4.6. Theorem. Let $F$ be an infinite-dimensional UHF algebra and let $A$ be a unital $C^{*}$-algebra with at most one tracial state. Suppose either

(1) $\operatorname{Inv}_{0}(A)$ is dense in $A$, or

(2) $A$ is simple, $K_{1}(A)=0$, and $A$ has a tracial state.

Then the $C^{*}$-tensor product $F \otimes A$ has the approximate positive factorization property.

Proof. Let $C=F \otimes A$. Write $F=\lim M_{k(n)}$ (with unital maps), and set $C_{n}=$ $M_{k(n)} \otimes A$. Thus $F \otimes A=\underline{\lim } C_{n}$. In case $(1), \operatorname{Inv}_{0}\left(C_{n}\right)$ is dense in $C_{n}$ by Lemma 
2.3, and it follows that $\operatorname{Inv}_{0}(C)$ is dense in $C$. In case $(2), C$ has stable rank 1 by Corollary 6.6 of [26]. Also, $K_{1}(C)=0$, so $\operatorname{Inv}_{0}(C)=\operatorname{Inv}(C)$ by Theorem 2.10 of [25]. Therefore $\operatorname{Inv}_{0}(C)$ is dense in $C$. Thus, in either case, $\operatorname{Inv}_{0}(C)$ is dense in C.

In both cases, each $C_{n}$ again has at most one tracial state, so the same holds for $C$, and Lemma 4.2 implies that $[C, C]+\mathbf{C} 1=C$. Lemma 4.3 now implies that for all $c \in C$ there is $\lambda \in \mathbf{T}$ such that $\lambda c \in P(C)^{-}$. To prove the theorem, it remains only to prove that $\lambda^{-1}$ is in $P(C)^{-}$. But it follows from Proposition 2.6(1) and (3) that $\operatorname{rank}(F)=\infty$, whence also $\operatorname{rank}(C)=\infty$. Thus, $\mathbf{T} \cdot 1 \subset P(C)^{-}$, and the theorem follows.

4.7. Example. In part (1) of the previous theorem, we can take $A$ to be $\mathbf{C}$ (giving a fast proof that infinite-dimensional UHF algebras have the approximate positive factorization property, a special case of Quinn's results as stated in Theorem 4.1 above), a type $\mathrm{II}_{1}$ factor, or the unitization of any stable $\mathrm{C}^{*}$-algebra $B$ satisfying $K_{1}(B)=0$ and $\operatorname{sr}(B)=1$. In part $(2)$, we can take $A$ to be the Choi algebra $C_{\mathrm{r}}^{*}(\mathbf{Z} / 2 \mathbf{Z} * \mathbf{Z} / 3 \mathbf{Z})$. (See Example (2) in Section 3 of $[6]$ and Theorem 2.8 (and its proof) in [5] for verification that the hypotheses of (2) are satisfied.)

We note that Theorem 7.2 of [27] shows that the algebras $F \otimes A$ in (2) have real rank zero whenever every quasitrace is a trace. This happens for all exact $\mathrm{C}^{*}$-algebras [11], and thus in particular for all nuclear $\mathrm{C}^{*}$-algebras.

We are now going to give necessary and sufficient conditions for an infinitedimensional simple direct limit $A$ with slow dimension growth as in [2] to have the approximate positive factorization property. Recall that slow dimension growth means that $A$ is a unital direct $\operatorname{limit} \lim A_{i}$, in which each $A_{i}$ is of the form $A_{i}=$ $\bigoplus_{t=1}^{s(i)} C\left(X_{i t}\right) \otimes M_{n(i, t)}$ for finite-dimensional connected compact Hausdorff spaces $X_{i t}$, such that

$$
\lim _{i \rightarrow \infty} \sup _{1 \leq t \leq s(i)} \operatorname{dim}\left(X_{i t}\right) / n(i, t)=0 .
$$

It is implicitly assumed (but not stated) in [2] that the algebras are supposed to be infinite-dimensional. Note (see [2]) that if $A$ is as above, and is simple, then

$$
\lim _{i \rightarrow \infty} \inf _{1 \leq t \leq s(i)} n(i, t)=\infty .
$$

The following lemma will be needed.

4.8. Lemma. For every $\varepsilon>0$ there is $N \in \mathbf{N}$ such that whenever $n \geq N$ and $\lambda_{1}, \ldots, \lambda_{n} \in \mathbf{T}$, then there exist $\mu_{1}, \ldots, \mu_{n} \in \mathbf{T}$ with $\mu_{1} \cdots \mu_{n}=1$ and such that the set $\left\{\mu_{1}^{-1} \lambda_{1}, \ldots, \mu_{n}^{-1} \lambda_{n}\right\}$ is $\varepsilon$-dense in $\mathbf{T}$. (That is, given $\lambda \in \mathbf{T}$, there is $k$ such that $\left|\lambda-\mu_{k}^{-1} \lambda_{k}\right|<\varepsilon$.)

Proof. Choose $m$ such that the primitive $m$-th root of 1 given by $\zeta=\exp (2 \pi i / m)$ satisfies $|\zeta-1|<\varepsilon / 2$. Let $N=m^{2}$. Let $n \geq N$ and let $\lambda_{1}, \ldots, \lambda_{n} \in \mathbf{T}$. The points $1, \zeta, \ldots, \zeta^{m-1}$ divide $\mathbf{T}$ into $m$ arcs, and it follows that one of these arcs, say the one from $\zeta^{k}$ to $\zeta^{k+1}$, must contain at least $m$ of the points $\lambda_{j}$. Renumbering, we may assume that $\lambda_{1}, \ldots, \lambda_{m}$ are in this arc. Set $\mu_{1}=1, \mu_{2}=\zeta, \ldots, \mu_{m}=\zeta^{m-1}$, and let $\mu_{m+1}, \ldots, \mu_{n}$ be arbitrary elements of $\mathbf{T}$ satisfying $\mu_{m+1} \cdots \mu_{n}=\zeta^{-m(m-1) / 2}$. Then $\left|\mu_{j}^{-1} \lambda_{j}-\zeta^{k-j+1}\right|<\varepsilon / 2$ for $1 \leq j \leq m$, and $\left\{\zeta^{k}, \zeta^{k-1}, \ldots, \zeta^{k-m+1}\right\}$ is $\varepsilon / 2$ dense in $\mathbf{T}$, so $\left\{\mu_{1}^{-1} \lambda_{1}, \ldots, \mu_{n}^{-1} \lambda_{n}\right\}$ is $\varepsilon$-dense. 
4.9. Theorem. Let $A$ be an infinite-dimensional simple unital direct limit with slow dimension growth as in [2]. Then $A$ has the approximate positive factorization property if and only if $A$ has real rank zero and $K_{1}(A)=0$.

Proof. The condition $K_{1}(A)=0$ is necessary by Theorem 2.4(2). To see that real rank zero is necessary, first note that $K_{0}(A)$ distinguishes the tracial states by Theorem 2.9. The Riesz decomposition property (Theorem 2.7 of [10]) then shows that the projections in $A$ distinguish the tracial states. Theorem 2 of [2] now implies that $A$ has real rank zero.

To prove sufficiency, note that Theorem 1 of [2] implies that $\operatorname{sr}(A)=1$. Theorem 2.10 of $[25]$ therefore implies that $\operatorname{Inv}(A) / \operatorname{Inv}_{0}(A) \rightarrow K_{1}(A)$ is an isomorphism. Thus, the hypotheses imply that $\operatorname{Inv}_{0}(A)$ is dense in $A$.

By polar decomposition, it now suffices to show that $U_{0}(A) \subset P(A)^{-}$. Write $A=\underline{\lim } A_{i}$, where each $A_{i}$ is of the form $A_{i}=\bigoplus_{t=1}^{s(i)} C\left(X_{i t}\right) \otimes M_{n(i, t)}$, in such a way that the system has slow dimension growth and all the maps are unital. Let $\varphi_{i}: A_{i} \rightarrow A$ and $\varphi_{i_{1}, i_{2}}: A_{i_{1}} \rightarrow A_{i_{2}}$ be the associated maps. Without loss of generality we may assume that the map from each $C\left(X_{i t}\right) \otimes M_{n(i, t)}$ to $A$ is nonzero. Let $u \in U_{0}(A)$ and let $\varepsilon>0$.

The first thing we have to do is ensure that we are working with elements with large spectrums. Choose $n_{0}$ to be the $N$ that works in the previous lemma for $\varepsilon / 8$ in place of $\varepsilon$. Choose $i_{0}$ so large that some $n\left(i_{0}, t_{0}\right)$ is at least $n_{0}$ and there is $u_{0} \in$ $U_{0}\left(A_{i_{0}}\right)$ with $\left\|u-\varphi_{i_{0}}\left(u_{0}\right)\right\|<\varepsilon / 8$. The kernel of the map from $C\left(X_{i_{0}, t_{0}}\right) \otimes M_{n\left(i_{0}, t_{0}\right)}$ to $A$ has the form $C_{0}(V) \otimes M_{n\left(i_{0}, t_{0}\right)}$ for some proper open subset $V \subset X_{i_{0}, t_{0}}$. Let $x_{0} \in X_{i_{0}, t_{0}}$ be any point not in $V$. Then $u_{0}\left(x_{0}\right)$ makes sense as an element of $M_{n\left(i_{0}, t_{0}\right)}$, and in a suitable basis can be written as $u_{0}\left(x_{0}\right)=\operatorname{diag}\left(\lambda_{1}, \ldots, \lambda_{n\left(i_{0}, t_{0}\right)}\right)$. Choose $\mu_{1}, \ldots, \mu_{n\left(i_{0}, t_{0}\right)}$ according to the conclusion of the previous lemma. Define $v_{0} \in U\left(A_{i_{0}}\right)$ to be the constant function 1 on $X_{i_{0}, t}$ for $t \neq t_{0}$, and the constant function $\operatorname{diag}\left(\mu_{1}, \ldots, \mu_{n\left(i_{0}, t_{0}\right)}\right)$ on $X_{i_{0}, t_{0}}$. Since $\operatorname{det}\left(\operatorname{diag}\left(\mu_{1}, \ldots, \mu_{n\left(i_{0}, t_{0}\right)}\right)\right)=1$, Ballantine's theorem [1] implies that $v_{0}$ is a product of positive elements. Set $w_{0}=v_{0}^{*} u_{0}$. By considering the value at $x_{0} \in X_{i_{0}, t_{0}}$, and noting that $x_{0} \notin V$, we see that $\operatorname{sp}\left(\varphi_{i_{0}}\left(w_{0}\right)\right)$ is $\varepsilon / 8$-dense in $\mathbf{T}$.

Clearly $\varphi_{i_{0}}\left(w_{0}\right) \in U_{0}(A)$. Since $A$ has real rank zero, it follows from Corollary 6 of [16] that there is $w_{1} \in U_{0}(A)$ with finite spectrum and $\left\|w_{1}-\varphi_{i_{0}}\left(w_{0}\right)\right\|<$ $\varepsilon / 8$. Thus there are distinct $\lambda_{1}, \ldots, \lambda_{m} \in \mathbf{T}$ (not the same as in the previous paragraph) and nonzero mutually orthogonal projections $p_{1}, \ldots, p_{m} \in A$ such that $w_{1}=\sum_{j=1}^{m} \lambda_{j} p_{j}$. We take the $\lambda_{j}$ to be ordered cyclically. The estimates above imply that $\operatorname{sp}\left(w_{1}\right)$ is $\varepsilon / 4$-dense in $\mathbf{T}$, and it follows that $\left|\lambda_{j}-\lambda_{j+1}\right|<\varepsilon / 2$ for $1 \leq j \leq m-1$.

Standard functional calculus arguments yield $i_{1}$ and mutually orthogonal projections $q_{1}, \ldots, q_{m} \in A_{i_{1}}$ (necessarily nonzero) such that the unitary $w_{2}=$ $\sum_{j=1}^{m} \lambda_{j} q_{j}$ satisfies $\left\|\varphi_{i_{1}}\left(w_{2}\right)-w_{1}\right\|<\varepsilon / 8$. Choose $N$ such that $2 \pi / N<\varepsilon / 8$, and use Lemma $\mathrm{F}$ of [2] to choose $i \geq i_{1}$ such that for every $t$ the image $q_{j}^{(t)}$ of $\varphi_{i_{1}, i}\left(q_{j}\right)$ in $C\left(X_{i t}\right) \otimes M_{n(i, t)}$ has rank at least $N+\operatorname{dim}\left(X_{i t}\right)$. (Note that this rank is constant on $X_{i t}$, since $X_{i t}$ is assumed connected.)

For each fixed $t$ we now construct, by induction on $j$, mutually orthogonal projections $e_{j}^{(t)} \in C\left(X_{i t}\right) \otimes M_{n(i, t)}$ which are trivial in the sense that each is Murray-von Neumann equivalent to a constant projection, and satisfying $e_{1}^{(t)} \leq q_{1}^{(t)}$, 
$e_{j}^{(t)} \leq q_{j-1}^{(t)}+q_{j}^{(t)}$ for $j \geq 2, \operatorname{rank}\left(e_{j}^{(t)}\right) \geq N$ for all $j$, and $\sum_{j=1}^{m} e_{j}^{(t)}=1$. To make the induction work, we impose the additional conditions

$$
\sum_{l=1}^{j} e_{l}^{(t)} \geq \sum_{l=1}^{j-1} q_{l}^{(t)} \quad \text { and } \quad \operatorname{rank}\left(\sum_{l=1}^{j} q_{l}^{(t)}-\sum_{l=1}^{j} e_{l}^{(t)}\right) \leq \operatorname{dim}\left(X_{i t}\right) / 2 .
$$

The existence of $e_{1}^{(t)}$ is immediate from Theorem 2.5(a) of [10]. Suppose now that $e_{1}^{(t)}, \ldots, e_{j}^{(t)}$ have been constructed; we construct $e_{j+1}^{(t)}$. If $j+1<m$, set $f=\sum_{l=1}^{j} q_{l}^{(t)}-\sum_{l=1}^{j} e_{l}^{(t)}$. Theorem 2.5(c) of [10] implies that $f$ is Murray-von Neumann equivalent to a subprojection of a trivial projection $g$ of rank at most $\operatorname{rank}(f)+\left(\operatorname{dim}\left(X_{i t}\right)-1\right) / 2 \leq \operatorname{dim}\left(X_{i t}\right)$. We may clearly assume $f \leq g$. By the same theorem, $g-f$ is Murray-von Neumann equivalent to a subprojection of $q_{j+1}^{(t)}$. Thus, we may assume $f \leq g \leq f+q_{j+1}^{(t)}$. Part (a) of this theorem now yields a trivial subprojection $h$ of $f+q_{j+1}^{(t)}-g$ such that

$$
\operatorname{rank}\left(f+q_{j+1}^{(t)}-(g+h)\right) \leq \operatorname{dim}\left(X_{i t}\right) / 2 .
$$

We take $e_{j+1}^{(t)}=g+h$. Note that

$$
\begin{aligned}
\operatorname{rank}\left(e_{j+1}^{(t)}\right) & \geq \operatorname{rank}(f)+\operatorname{rank}\left(q_{j+1}^{(t)}\right)-\operatorname{dim}\left(X_{i t}\right) / 2 \\
& \geq \operatorname{rank}\left(q_{j+1}^{(t)}\right)-\operatorname{dim}\left(X_{i t}\right) \geq N .
\end{aligned}
$$

This completes the induction step in case $j+1<m$. If $j+1=m$, then set $e_{j+1}^{(t)}=1-\sum_{l=1}^{m-1} e_{l}^{(t)}$. Note that

$$
\operatorname{rank}\left(e_{j+1}^{(t)}\right) \geq N+\operatorname{dim}\left(X_{i t}\right) \geq \operatorname{dim}\left(X_{i t}\right) / 2,
$$

and the sum of $e_{j+1}^{(t)}$ and the trivial projection $\sum_{l=1}^{m-1} e_{l}^{(t)}$ is the trivial projection 1 , so that Theorem 2.5(b) of [10] implies that $e_{j+1}^{(t)}$ is trivial. This completes the induction.

We now define $e_{j}=\sum_{t} e_{j}^{(t)}$ and $w_{3}=\sum_{j} \lambda_{j} e_{j}$. Since $e_{j} \leq q_{j-1}+q_{j}$, and since $\left|\lambda_{j}-\lambda_{j-1}\right|<\varepsilon / 2$, we have $\left\|w_{3}-\varphi_{i_{1}, i}\left(w_{2}\right)\right\|<\varepsilon / 2$. We will now approximate $w_{3}$ by a product of positive elements. For each $j$ and $t$, let $N_{j}^{(t)}=\operatorname{rank}\left(e_{j}^{(t)}\right) \geq N$, and let $\alpha_{j}^{(t)}$ by the least nonnegative real number such that $\left[\exp \left(2 \pi i \alpha_{j}^{(t)}\right) \lambda_{j}\right]^{N_{j}^{(t)}}=1$. Note that $0 \leq \alpha_{j}^{(t)} \leq 1 / N$, so that $\left|\exp \left(2 \pi i \alpha_{j}^{(t)}\right)-1\right| \leq 2 \pi / N$. Now define $w_{4}=$ $\sum_{j, t} \exp \left(2 \pi i \alpha_{j}^{(t)}\right) \lambda_{j} e_{j}^{(t)}$. Then $w_{4}$ is a unitary which satisfies $\left\|w_{4}-w_{3}\right\| \leq 2 \pi / N<$ $\varepsilon / 8$. Furthermore, for each $j$ and $t$, the choice of $\alpha_{j}^{(t)}$ and the fact that $e_{j}^{(t)}$ is a trivial projection imply that $\exp \left(2 \pi i \alpha_{j}^{(t)}\right) \lambda_{j} e_{j}^{(t)}$ is a constant scalar multiple of the identity in

$$
e_{j}^{(t)}\left(C\left(X_{i t}\right) \otimes M_{n(i, t)}\right) e_{j}^{(t)} \cong C\left(X_{i t}\right) \otimes M_{N_{j}^{(t)}}
$$

whose determinant is 1 . Using Ballantine's theorem on these elements and forming the direct sum over $j$ and $t$, we therefore see that $w_{4}$ is a product of five positive elements. 
We now have $\left\|u-\varphi_{i_{0}}\left(u_{0}\right)\right\|<\varepsilon / 8, \quad u_{0}=v_{0} w_{0}$, and

$$
\begin{aligned}
\left\|\varphi_{i_{0}}\left(w_{0}\right)-\varphi_{i}\left(w_{4}\right)\right\| \leq & \left\|\varphi_{i_{0}}\left(w_{0}\right)-w_{1}\right\|+\left\|w_{1}-\varphi_{i_{1}}\left(w_{2}\right)\right\| \\
& +\left\|\varphi_{i_{1}, i}\left(w_{2}\right)-w_{3}\right\|+\left\|w_{3}-w_{4}\right\| \\
& <\varepsilon / 8+\varepsilon / 8+4 \varepsilon / 8+e / 8=7 \varepsilon / 8
\end{aligned}
$$

furthermore, both $v_{0}$ and $w_{4}$ are finite products of positive elements. Thus $u$ can be approximated to within $\varepsilon$ by finite products of positive elements, and the proof that $U_{0}(A) \subset P(A)^{-}$is complete.

This proof actually shows that products of 11 positive invertible elements are dense (one from the polar decomposition and five from each application of Ballantine's theorem).

The proof of this theorem also implies the following result, which holds regardless of whether the algebra has real rank zero or one.

4.10. Theorem. Let $A$ be an infinite-dimensional simple unital direct limit with slow dimension growth as in [2]. Then the rank of $A$ is infinite.

Proof. If the element $u$ in the previous proof is a scalar multiple of the identity, then the unitary $\varphi_{i_{0}}\left(w_{0}\right)$ constructed in the proof will already have finite spectrum. The only use of real rank zero in that direction of the proof was to replace this unitary with a nearby one with finite spectrum.

4.11. Example. Let $X$ be a finite CW complex such that $K^{0}(X)$ has a nontrivial torsion element $\eta$ and $K^{1}(X)=0$. Choose a sequence $x_{1}, x_{2}, \ldots$ in $X$ such that each tail of the sequence is dense in $X$. Define $A_{i}=C\left(X, M_{2^{i}}\right)$ and define $\varphi_{i, i+1}: A_{i} \rightarrow$ $A_{i+1} \cong M_{2}\left(A_{i}\right)$ by $\varphi_{i, i+1}(f)=\operatorname{diag}\left(f, f\left(x_{i}\right) \cdot 1\right)$. It follows from [9] that $A=\lim A_{i}$ is simple and has real rank zero, and one computes directly that $K_{1}(A)=\overrightarrow{0}$ and that the image of $\eta \in K_{0}\left(A_{0}\right)$ in $K_{0}(A)$ is nonzero. Therefore $A$ is a separable simple nuclear non-AF algebra, which has the approximate positive factorization property by Theorem 4.9 .

A simple example of such a finite CW complex $X$ can be constructed as follows. Let $D \subset \mathbf{C}$ be the closed unit disk. Let $\sim$ be the equivalence relation $z_{1} \sim z_{2}$ if $\left|z_{1}\right|=\left|z_{2}\right|=1$ and $z_{1}^{n}=z_{2}^{n}$. Then $X=D / \sim$ is a finite CW complex such that $K^{0}(X) \cong \mathbf{Z} \oplus \mathbf{Z} / n \mathbf{Z}$ and $K^{1}(X)=0$.

Theorem 4.9 raises the following two questions.

4.12. Question. Let $A$ be a simple infinite-dimensional unital $\mathrm{C}^{*}$-algebra with real rank zero, stable rank one, and $K_{1}(A)=0$. Does it follow that $A$ has the approximate positive factorization property?

Theorem 4.9 shows that the answer is yes for infinite-dimensional direct limits with slow dimension growth. The answer is also yes if $A$ has infinite rank and a unique tracial state, by Lemmas 4.2 and 4.3 .

4.13. Question. Let $A$ be a simple unital $\mathrm{C}^{*}$-algebra with the approximate positive factorization property. Does it follow that $A$ has real rank zero?

Theorem 4.9 shows that the answer to this question is also yes for infinitedimensional direct limits with slow dimension growth. Furthermore, Theorem 2.9 
and Proposition 2.13 imply that if $A$ is a unital algebra with the approximate positive factorization property, then at least the stabilization $K \otimes A$ has a fair number of projections - enough to distinguish the tracial states, and, if there is a tracial state $\tau$, then enough that $\tau_{*}\left(K_{0}(A)\right)$ is dense in $\mathbf{R}$. The requirements that $A$ be unital and simple are necessary, as is shown by the next theorem and by Example 4.16.

We now give necessary and sufficient conditions for a stable $\mathrm{C}^{*}$-algebra to have the approximate positive factorization property.

4.14. Theorem. Let $A$ be any $C^{*}$-algebra. Then $K \otimes A$ has the approximate positive factorization property if and only if $\operatorname{sr}(A)=1$ and $K_{1}(A)=0$.

Proof. Let $B=K \otimes A$.

If $B$ has the approximate positive factorization property, then $\operatorname{sr}(A)=1$ by Theorem 2.4(3) and Theorem 3.6 of [24], and $K_{1}(A)=0$ by Theorem 2.4(2).

For the converse, let $\operatorname{sr}(A)=1$ and $K_{1}(A)=0$, so that $\operatorname{sr}(B)=1$ and $K_{1}(B)=0$. Then $\operatorname{Inv}_{0}(\tilde{B})$ is dense in $\tilde{B}$, and it easily follows that $\operatorname{Inv}_{0}(B)$ is dense in $B+1$. Next, note that $[\tilde{B}, \tilde{B}]=B$ by Lemma 4.2 . Lemma 3.2 then implies that $\exp (b) \in$ $P(B+1)^{-}$for all $b \in B$. Since every element of $\operatorname{Inv}_{0}(B)$ is a product of exponentials $\exp (b)$ with $b \in B$, it follows that $B+1=\operatorname{Inv}_{0}(\tilde{B})^{-} \subset P(B+1)^{-}$.

We note that it is shown in [15] that every element of $\operatorname{Inv}_{0}(K \otimes A)$ is a product of finitely many positive invertible elements of $(K \otimes A)^{-}$. Clearly we may restrict these elements to be in $(K \otimes A)+1$. Thus, in the theorem above, if $\operatorname{sr}(A)=1$ and $K_{1}(A)=0$, then every invertible element of $(K \otimes A)+1$ is in fact exactly a product of positive elements.

4.15. Remark. This theorem, combined with Theorem 2.4, shows that if $A$ has the approximate positive factorization property, then so does $K \otimes A$. The converse is false, even for simple $\mathrm{C}^{*}$-algebras. It is easy to construct, using the methods of [9] (as in Example 4.11), an infinite-dimensional simple unital $\mathrm{C}^{*}$-algebra $A$, obtained as a direct limit with slow dimension growth, such that $A$ has trivial $K_{1}$ group, stable rank 1, and real rank 1. Theorem 4.14 implies that $K \otimes A$ has the approximate positive factorization property, while Theorem 4.9 implies that $A$ does not.

4.16. Example. We give an example of a unital $\mathrm{C}^{*}$-algebra $A$ with the approximate positive factorization property which contains an ideal $I$ without the approximate positive factorization property. This example shows that Theorem 3.6 can't be extended to say that the approximate positive factorization property passes to ideals, even when the quotient also has the approximate positive factorization property. Our algebra $A$ also does not have real rank zero, showing that in general the approximate positive factorization property does not imply real rank zero.

Set $I_{0}=K \otimes C(\mathbf{T})$. It follows from Kasparov's extension theory [13] and the Universal Coefficient Theorem (Theorem 1.17 of [28]) that there is an extension

$$
0 \rightarrow I_{0} \rightarrow A_{0} \rightarrow \mathbf{C} \rightarrow 0
$$

such that the induced map from $K_{0}(\mathbf{C})$ to $K_{1}\left(I_{0}\right)$ is an isomorphism. (Note that $A_{0}$ will not be unital.) The long exact sequence in $K$-theory then shows that $K_{1}\left(A_{0}\right)=$ 0 , and Theorem 4.11 of [24] shows that $\operatorname{sr}\left(A_{0}\right)=1$. (Note that $\operatorname{sr}\left(I_{0}\right)=1$.) So 
$K \otimes A_{0}$ has the approximate positive factorization property by Theorem 4.14 . Its ideal $K \otimes I_{0}$ does not have the approximate positive factorization property, because $K_{1}\left(K \otimes I_{0}\right) \neq 0$. Furthermore, $K \otimes A_{0}$ does not have real rank zero, because it contains the ideal $K \otimes I_{0}$ whose real rank is not zero. (See [3].)

The algebra $K \otimes A_{0}$ is not unital, but it can be used to construct a unital example $B$ in the following two ways. Either unitize it, and then form the tensor product with an infinite dimensional UHF algebra $F$, or form a unital trivial extension by an infinite dimensional UHF algebra.

In the second case, $B$ will have the approximate positive factorization property by Theorem 3.6(1), and will still contain the ideal $K \otimes I_{0}$ which has neither the approximate positive factorization property nor real rank zero. Since this ideal does not have real rank zero, neither does $B$. In the first case, $B$ will have the approximate positive factorization property by Theorem 4.6(1). It will now contain the ideal $F \otimes K \otimes I_{0} \cong F \otimes K \otimes K \otimes C(\mathbf{T})$. Since this ideal has nontrivial $K_{1}$, it does not have the approximate positive factorization property. Cutting it down by a rank one projection in $K \otimes K$, we obtain a hereditary subalgebra of $B$ which is isomorphic to $F \otimes C(\mathbf{T})$. This algebra does not have real rank zero because the projections don't distinguish the traces. It follows from Corollary 2.8 of [3] that $B$ does not have real rank zero either.

\section{REFERENCES}

1. C. S. Ballantine, Products of positive definite matrices, IV, Linear Alg. Appl. 3 (1970), 79-114. MR 41:1766

2. B. Blackadar, M. Dădărlat, and M. Rørdam, The real rank of inductive limit $C^{*}$-algebras, Math. Scand. 69 (1991), 211-216. MR 93e:46067

3. L. G. Brown and G. K. Pedersen, $C^{*}$-algebras of real rank zero, J. Funct. Anal. 99 (1991), 131-149. MR 92m:46086

4. H. Choda, An extremal property of the polar decomposition in von Neumann algebras, Proc. Japan Acad. 46 (1970), 341-344. MR 50:8098

5. M.-D. Choi, A simple $C^{*}$-algebra generated by two finite-order unitaries, Canadian J. Math. 31 (1979), 867-880. MR 80j:46092

6. J. Cuntz, K-theoretic amenability for discrete groups, J. Reine Ang. Math. 344 (1983), 180195. MR 86e: 46064

7. J. Cuntz and G. K. Pedersen, Equivalence and traces on $C^{*}$-algebras, J. Funct. Anal. 33 (1979), 135-164. MR 80m:46053

8. P. de la Harpe and G. Skandalis, Déterminant associé à une trace sur une algèbre de Banach, Ann. Inst. Fourier 43-1 (1984), 241-260. MR 87i:46146a

9. K. R. Goodearl, Notes on a class of simple $C^{*}$-algebras with real rank zero, Publ. Sec. Mat. Univ. Autónoma Barcelona 36 (1992), 637-654. MR 94f:46092

10. K. R. Goodearl, Riesz decomposition in inductive limit $C^{*}$-algebras, Rocky Mountain J. Math. 24 (1994), 1405-1430. CMP 95:9

11. U. Haagerup, Quasitraces on exact $C^{*}$-algebras are traces, (1991) (handwritten manuscript).

12. P. R. Halmos, A Hilbert Space Problem Book, Springer, New York 1982. MR 84e:47001

13. G. G. Kasparov, The operator K-functor and extensions of $C^{*}$-algebras, Izv. Akad. Nauk SSSR Ser. Mat. 44 (1980), 571-636; English transl., Math. USSR Izv. 16 (1981), 513-572. MR 81m:58075

14. M. Khalkhali, C. Laurie, B. Mathes, and H. Radjavi, Approximation by products of positive operators, J. Operator Theory 29 (1993), 237-247. CMP 94:15

15. M. Leen, Factorization in the Invertible Group of a $C^{*}$-Algebra, Ph.D. thesis, University of Oregon, Eugene, OR, 1994.

16. H. Lin, Exponential rank of $C^{*}$-algebras with real rank zero and Brown-Pedersen's conjecture, J. Funct. Anal. 114 (1993), 1-11. MR 95a:46079 
17. G. J. Murphy, The analytic rank of a $C^{*}$-algebra, Proc. Amer. Math. Soc. 115 (1992), 741746. MR 92i:46085

18. C. Pearcy and D. M. Topping, Sums of small numbers of idempotents, Michigan J. Math. 14 (1967), 453-465. MR 36:2006

19. N. C. Phillips, Simple $C^{*}$-algebras with the property weak (FU), Math. Scand. 69 (1991), 127-151. MR 93d:46121

20. N. C. Phillips, Exponential length and traces, Proc. Royal Soc. Edinburgh, Sec. A 125 (1995), 13-29. CMP 95:8

21. N. C. Phillips, Factorization problems in the invertible group of a homogeneous $C^{*}$-algebra, (preprint) University of Oregon, 1993.

22. T. Quinn, Ideals in AF-algebras and approximation by products of positive operators, preprint, Trinity College, Dublin, 1993.

23. H. Radjavi, Products of Hermitian matrices and symmetries, Proc. Amer. Math. Soc. 21 (1969), 369-372; 26 (1970), 701. MR 39:1470

24. M. A. Rieffel, Dimension and stable rank in the K-theory of $C^{*}$-algebras, Proc. London Math. Soc. 46 (1983), no. (3), 301-333. MR 84g:46085

25. M. A. Rieffel, The homotopy groups of the unitary groups of noncommutative tori, J. Operator Theory 17 (1987), 237-254. MR 88f:22018

26. M. Rørdam, On the structure of simple $C^{*}$-algebras tensored with a UHF algebra, J. Funct. Anal. 100 (1991), 1-17. MR 92m:46091

27. M. Rørdam, On the structure of simple $C^{*}$-algebras tensored with a UHF algebra, II, J. Funct. Anal. 107 (1992), 255-269. MR 93f:46094

28. J. Rosenberg and C. Schochet, The Künneth theorem and the universal coefficient theorem for Kasparov's generalized K-functor, Duke Math. J. 55 (1987), 431-474. MR 88i:46091

29. P. Y. Wu, Products of normal operators, Canadian J. Math. 40 (1988), 1322-1330. MR 90d: 47039

Department of Mathematics, University College, Cork, Ireland

E-mail address: gjm@ucc.ie

Department of Mathematics, University of Oregon, Eugene, Oregon 97403-1222

E-mail address: phillips@bright.uoregon.edu 